\section{TIA PREHOSPITAL REFERRAL FEASIBILITY TRIAL (TIER): RECRUITMENT AND INTERVENTION USAGE}

${ }^{1} \mathrm{~N}$ Rees, ${ }^{2} \mathrm{C}$ Hampton*, ${ }^{2} \mathrm{~s}$ Bulger, ${ }^{3} \mathrm{~K}$ Ali, ${ }^{4} \mathrm{~T}$ Quinn, ${ }^{5} \mathrm{G}$ Ford, ${ }^{2}$ Ashley Akbari, ${ }^{6}$ Matthew Ward, ${ }^{2}$ Alison Porter, ${ }^{1} \mathrm{C}$ Jones, ${ }^{2} \mathrm{H}$ Snooks. ${ }^{1}$ Welsh Ambulance Service Trust (WAST); ${ }^{2}$ Swansea University; ${ }^{3}$ Brighton and Sussex Medical School; ${ }^{4}$ Kingston University and Saint George's University; ${ }^{5}$ Oxford Academic Health Science Network; ${ }^{6}$ West Midlands Ambulance Service

\subsection{6/10.1136/bmjopen-2018-EMS.73}

Aim Early specialist assessment of Transient Ischaemic Attack (TIA) can reduce the risk of stroke and death. We assessed feasibility of undertaking a multi-centre cluster randomised trial to evaluate clinical and cost effectiveness of referral of patients attended by emergency ambulance paramedic with low-risk TIA directly to specialist TIA clinic for early review. Method We randomly allocated volunteer paramedics to intervention or control group. Intervention paramedics were trained to deliver the intervention during the patient recruitment period. Control paramedics continued to deliver care as usual. Patients with TIA were identified from hospital records. Results Development and recruitment phases are complete, with outcome follow up ongoing. Eighty nine of 134 (66\%) paramedics participated in TIER. Of 1377 patients attended by trial paramedics during the patient recruitment period, 53 $(3.8 \%)$ were identified as eligible for trial inclusion. Three of $36(8 \%)$ patients attended by intervention paramedics were referred to the TIA clinic. Of the others, only one appeared to be a missed referral; in one case there was no prehospital record of TIA; one was attended by a paramedic who was not TIER trained; one patient record was missing; all others were recorded with contraindications: FAST positive $(n=13)$; ABCD2 score $>3(n=5)$; already taking warfarin $(n=2)$; crescendo TIA $(n=1)$ other clinical factors $(n=8)$.

Conclusion Preliminary results indicate challenges in recruitment and low referral rates. Further analyses will focus on whether progression criteria for a definitive trial were met, and clinical outcomes from this feasibility trial.

Conflict of interest None

Funding None

\section{PARAMEDICS' EXPERIENCES OF ADMINISTERING FASCIA ILIACA COMPARTMENT BLOCK TO PATIENTS WITH SUSPECTED HIP FRACTURE}

${ }^{1} \mathrm{~B}$ Evans*, ${ }^{2} \mathrm{~A}$ Brown, ${ }^{1} \mathrm{~J}$ Bulger, ${ }^{1} \mathrm{G}$ Fegan, ${ }^{3} \mathrm{~S}$ Ford, ${ }^{3} \mathrm{~K}$ Guy, ${ }^{2} \mathrm{~S}$ Jones, ${ }^{4} \mathrm{~L}$ Keen, ${ }^{3} \mathrm{I}$ Pallister, ${ }^{4} \mathrm{~N}$ Rees, ${ }^{1} \mathrm{I}$ Russell, ${ }^{1} \mathrm{~A}$ Seagrove, ${ }^{1} \mathrm{H}$ Snooks. ${ }^{1}$ Swansea University Medical School (SUMS); ${ }^{2}$ Patient representatives; ${ }^{3}$ Abertawe Bro Morgannwg University Health Board (ABMU); ${ }^{4}$ Welsh Ambulance Services NHS Trust (WAST)

\subsection{6/10.1136/bmjopen-2018-EMS.74}

Aim Pre-hospital pain management for hip fracture is inadequate, with risk of complications, particularly from morphine. Fascia Iliaca Compartment Block (FICB) is used in hospital. The RAPID trial tested feasibility of paramedics administering FICB to patients with suspected hip fracture at the scene of injury.

Method We held three focus groups with 11 paramedics serving one hospital, audio-recorded with participants' consent. Two researchers, one paramedic and one lay member conducted thematic analysis of interview transcripts.

Results Respondents believed FICB was a suitable intervention for paramedics to deliver, aligning with their routine practice and within people's capabilities to administer. They said it took up to 10 min longer than usual care to deliver, in part due to nervousness and unfamiliarity with a new procedure. They praised the training provided but said they were anxious about causing harm by injecting into the wrong location. Reported challenges related to the emergency context: patients often waited many hours for ambulance arrival; they sometimes needed to be moved from awkward locations which exacerbated pain; family and neighbours were present as paramedics administered treatment. Although uncertain whether FICB reduced patients' pain more effectively than other pain relief options, respondents believed it was safer for elderly people.

Conclusion Paramedics are willing and able to administer FICB to patients with suspected hip fracture before ambulance transport to hospital. Feasibility study findings will inform a research proposal for a definitive multi-centre trial of paramedic administered prehospital FICB.

Conflict of interest None

Funding None

\section{TRIAGE OF ELDERLY CITIZENS CALLING A DANISH MEDICAL HELPLINE}

\begin{abstract}
1,2S Miseljic*, ${ }^{1,3} \mathrm{H}$ Gamst-Jensen, ${ }^{1} \mathrm{~F}$ Folke, ${ }^{2,4} \mathrm{~T}$ Møller. ${ }^{1}$ Emergency Medical Services Copenhagen, University of Copenhagen, Denmark; ${ }^{2}$ University of Copenhagen, Denmark: ${ }^{3}$ The National Institute for Public Health, University of Southern Denmark, Copenhagen, Denmark; ${ }^{4}$ The University Hospitals Centre for Health Research, UCSF Copenhagen Denmark
\end{abstract}

\subsection{6/10.1136/bmjopen-2018-EMS.75}

Aim Symptom description and degree-of-worry (DOW) by elderly contacting a medical helpline are poorly described. ${ }^{1}$ Several diagnoses may be associated with preventable admissions for elderly citizens, ${ }^{2}$ and increased risk of over-and-under-triage. $^{3}$ We aimed to investigate how symptoms and worry are described by elderly citizens (age 65 and older) when they call a medical hotline.

Method A mixed method design on data gathered from a three-week 2017 cohort, where callers rated their DOW on a 1-5 scale. A sub-cohort of $65+$ years was used. The National Patient Register gave data on admissions. Calls made by the patients (patient-caller) was included for the qualitative analysis $(\mathrm{n}=90)$.

Results A total of 1530 acutely ill or injured elderly called the medical helpline. $\mathrm{n}=755 \quad(50 \%)$ were patient-callers and $\mathrm{n}=364(48 \%)$ of these had a high DOW. Of all patient-callers $\mathrm{n}=216(28.6 \%)$ were triaged to face-to-face consultation and $\mathrm{n}=73(9.7 \%)$ were subsequently admitted. The preliminary qualitative analysis led to the hypothesis that patient-callers often expressed exacerbation of chronic diseases (which are part of the preventable admission) and often lived alone. Those that were not triaged to face-to-face consultation were frequently offered one, but rejected because of the obstacles of leaving their home. The majority of patient-callers triaged to face-to-face consultation expressed that their general practitioner was not able to help them.

Conclusion The majority of the elderly patient-callers was very worried and lived alone. Most of the calls concerned exacerbation of a chronic condition and most of the symptoms fitted the described preventable admission diagnoses. 


\section{REFERENCES}

1. Gamst-Jensen $\mathrm{H}$, et al. Self-rated worry predicts hospitalisation in out-of-hours services telephone triage. Copenhagen; 2018. (abstact, not published).

2. KL. Regions of Denmark, Ministry of Finance and Ministry of Health, 2016; Indblik i sundhedsvæsenets resultater 2016, Report published by Ministry of Health. Avaliable at [cited 2018 Jan 12] http://www.sum.dk/ /media/Filer - Publikationer_i_pdf/2016/Indblik-i-sundhedsvaesenets-resultater-maj-2016/Indblik-i-sundhedsvaesenets-resultater-maj-2016.pdf

3. Gamst-Jensen H, Lippert FK, Egerod I. Under-triage in telephone consultation is related to non-normative symptom description and interpersonal communication: A mixed methods study. Scand J Trauma Resusc Emerg Med 2017 December $15 ; 25(1): 52$.

Conflict of interest None

Funding None

\section{A NATIONWIDE WEB-BASED QUALITY REGISTRY FOR DISPATCHER-ASSISTED CARDIOPULMONARY RESUSCITATION (DACPR) OF OUT-OF-HOSPITAL CARDIAC ARREST (OHCA) - AN INNOVATIVE STRUCTURED MEASUREMENT}

${ }^{1} \mathrm{PC}$ Ko* ${ }^{2} \mathrm{CC}$ Cheng, ${ }^{2} \mathrm{WL}$ Chen. ${ }^{1}$ National Taiwan University Hospital, Taiwan; ${ }^{2}$ National Fire Agency, Taiwan

\subsection{6/10.1136/bmjopen-2018-EMS.76}

Aim Following the guidelines of DACPR may enhance bystander CPR rate after OHCA. Registry of quality measurement for DACPR has never been explored. We designed a nationwide quality registry for DACPR performance and innovated a structured format of measurement.

Method A nationwide Google Forms based online registry covering over twenty administrative regions and more than twenty millions of population was designed and launched for DACPR performance and quality measurement at individual case level for non-traumatic OHCA patient. Audio records of individual EMS call were reviewed for performance rating.

Designs System data inputted could be immediately retrieved as feedback to each corresponding administrative region. Recognition of cardiac arrest by call communication, CPR Instructions upon the recognised OHCA, and chest compression upon the recognised OHCA were the three major categorical performance indicators, and each operational time interval of call-to-recognition, call-to-instruction, and call-to-compression were evaluated. Each categorical performance indicator ( $\mathrm{Y}$ axis) was paired with its operational time interval (X axis) as a set of quality index for diagrammatic comparison in our design. We used regression analysis for statistical analysis.

Results A total of 5642 audio records for OHCA EMS calls across 17 regions were centralised into the nationwide DACPR Quality Registry in 6 months (minimal 40 to maximal 1622 cases/region according to its population). Regional recognition rate significantly varied from $10.0 \%$ to $65.5 \%$ ( $p<0.01$; averaged $51.0 \%$, SD 20.0\%). Instruction rate varied from $41.3 \%$ to $95.0 \%(\mathrm{p}<0.01$; averaged $80.0 \%$, SD $28.5 \%)$. Compression rate varied from $0 \%$ to $87.5 \%(\mathrm{p}<0.01$; averaged $54.0 \%$, SD 23.6\%). Averaged regional call-to-recognition time, call-toinstruction time, and call-to-compression time were 48 (SD 19), 84 (SD 42), and 185 (SD 114) seconds. The designated diagrammatic comparisons may indicate the administrative regions of better performance located at the upward and leftward dimension, and the ones of unsatisfied performance located at the downward and rightward dimension (diagrams will be illustrated).
Conclusion We successfully innovated and launched a nationwide DACPR quality e-registry showing a wide variety of regional performance needing improvement. The designated diagram may easily indicate and compare the individual performance across the joint regions.

Conflict of interest None

Funding None

\section{OPIOID OVERDOSE DEATH IN WALES FROM 2012 TO 2015: A LINKED DATA AUTOPSY STUDY}

${ }^{1} \mathrm{M}$ Jones ${ }^{*},{ }^{1} \mathrm{H}$ Snooks, ${ }^{1} \mathrm{~A}$ Watkins, ${ }^{2} \mathrm{G}$ Fuller. ${ }^{1}$ Swansea University, UK; ${ }^{2}$ University of Sheffield, UK

10.1136/10.1136/bmjopen-2018-EMS.77

We performed a retrospective autopsy study to better understand factors associated with opioid poisoning death. Using anonymised linked data we describe demographic characteristics, emergency service utilisation, and clinical presentation prior to death.

Method Decedents of opioid poisoning in Wales in 2015 were identified from Office of National Statistics (ONS) mortality data and their records linked with the Emergency Department data.

Results Age at death ranged 18 to 78 years, with a mean of 42. Average male age was 41 and average female age was 44 . $76 \%$ of decedents were men $(n=98 / 112) .87 \%$ of decedents $(n=112 / 129)$ attended the emergency department in the three years prior to death; $\mathrm{n}=89$ in the previous year, 99 in the previous two years and 112 in the previous three years. $84 \%$ of male and $93 \%$ of female decedents attended the ED in the three years prior to death. There were 665 attendances, and half involved conveyance by ambulance. Attendances per individual ranged from 1 to 60 , with over half of decedents attending more than three times. Diagnostic codes were mostly missing or non-specific, with only $6.5 \%$ of attendances representing 27 decedents, coded as drug related.

Conclusion Matching previously published data, we found that fatal opioid poisoning is preceded by a period of high emergency health service utilisation. On average decedents were in their fifth decade and more likely to be male than female. Attendances varied widely, with men less likely to attend than women.

Conflict of interest None

Funding PRIME Centre Wales

\section{IMPACT OF ADDITIONAL CALL TRIAGE TIME ON EMS RESPONSE PERFORMANCE AND RESOURCE USE}

J Turner*, R Jacques. SHARR, University of Sheffield, Sheffield, UK

\subsection{6/10.1136/bmjopen-2018-EMS.78}

Aim Time based standards have been used as a key performance measure for EMS internationally but can lead to operational behaviours that are not clinically focussed. NHS England tested a new operational model (Dispatch on Disposition) allowing additional call triage time of up to $4 \mathrm{~min}$ before starting the response interval clock start.

Method A controlled before and after time series analysis of the intervention implemented in 6 of the 10 regional EMS services. We measured weekly trends in average resource 